\section{THERMODYNAMICS AND HUMANISM}

Time and Thermodynamics

By A. R. Ubbelohde. Pp. vii +110.

Oxford University Press, 1947.) 6s. net.

(London :

$\mathrm{T}$

HIS little book shows some confusion of aim. I do not think Prof. A. R. Ubbelohde decided clearly what kind of book he wanted to write. According to the preliminary chapter, his intention appears to have been to write on the wider human aspects of thermodynamies, to contribute to scientific humanism, which he defines as the unification of the specialized sciences from the point of view of their human interest. In this and in his explanations of the significance of entropy as providing a direction pointer for time, he is on fairly familiar ground, and though his treatment inevitably calls forth a comparison with the popular writings of Eddington, who covered similar territory with, one must say, considerably more force, he is both competent and interesting.

The body of the book is, however, a fairly straightforward explanation of the laws of thermodynamics, partly classical and partly statistical, which will undoubtedly be helpful to students of the physical sciences, since it will enable them to appreciate some of the more difficult parts of the subject. But the inquiring layman may be repelled by even simple algebra, and he will find the chapter on the second law, which does not differ in any essentials from that found in most text-books of thermodynamics, rather stiff going.

The discussion of the human significance of the laws of thermodynamics, being confined mainly to short chapters at the beginning and the end, is disappointing. Most readers looking for humanistic meanings will turn to the chapter on thermodynamics and life to see what the author has to say; but they will have some difficulty in determining what his attitude really is. He favours, without dogmatism, the very reasonable view that "living organisms are characterised thermodynamically not by any vital power of selection of individual molecules, but by the fact that the organism considered as a unit is continually effecting processes in which the entropy decreases, at the expense of rather greater compensating increases of entropy in the surround. ings".

So far, good. Few readers will be inclined to disagree with the author when he explains that living things are entirely unique arrangements for effecting locally such 'disentropic' processes, and quite unlike anything encountered in non-living matter. But what are we to make of the following: "Currently accepted terminology would describe human control over natural forces and human organisation of inanimate processes as intelligent. The organisation of similar processes within living organisms, which normally involves more complex integration than has yet been achieved through human inventiveness, must be the result of analogous intelligent inventiveness" (my italies) ? It would be of interest to have this argument developed, though it is not particularly new (vide Paley's watch); but the author puts off his readers with the remark "Full elaboration of the inventor analogy would not be possible without very greatly exceeding the scope of this book. The issues involved are profound."
If I understand it correctly, the argument is that although the chemical happenings in living organisms may be fully in accordance with the laws of thermodynamics, yet the existence of such extraordinarily complex arrangements for utilizing these laws to produce organisms capable of individual activities, and in many cases intelligence, involves something in addition.

Any fresh arguments for this view would be of great interest. What the author actually does is to mention certain lines of argument and dismiss them as outside the scope of the book. For example, he says, "A further point which may be mentioned, but which cannot be developed very far in this book, is that if the human intelligence is able to conceive (at least by negation) of conditions in which the laws of nature, such as the trend in entropy, are not obeyed, it cannot itself be wholly subject to these laws". Or again, "But there is no real promise of ever bridging the gap between the mechanism as a whole and the organism as a whole, through increased human skill. The nature of the difference between mechanism and organism cannot be further resolved without bringing in other considerations about life which lie outside the scope of this book." It is scarcely fair to the reader to raise such points and leave them up in the air. One cannot help wondering how much the author's final conclusion that "human living is part in entropy-time and part outside it" is based on the "other considerations".

I do not want to suggest that $I$ think there are no other considerations which are worthy of attention. They have been stated with varying degrees of cogency and charm by poets and philosophers as well as some men of science. But Prof. Ubbelohde is shy of developing his argument in this direction. He reminds us of the Scotsman's remark to a lady who had been praising the English: "But hae ye ever been able to get one of them into a metapheesical argument?" He refuses to be drawn into metaphysical arguments; but does not mind supporting his point of view by referring to their existence. It is rather curious that he never mentions, though he may imply, the one inevitable teleological conclusion from the second law, that if the world as we know it is steadily running down, there must have been a winding up.

J. A. V. Butler

\section{SCHOOL CERTIFICATE BIOLOGY}

\section{Essentials of Biology}

An Introductory Text-Book for Secondary Schools. By W. F. Wheeler. Pp. xi $+266+8$ plates. (London: William Heinemann, Ltd., 1947.) 7s. $6 d$.

A $\mathrm{S}$ was indicated in Nature a short time ago (159, A $147 ; 1947)$, in the teaching of biology a new movement is spreading through the schools, of considerable educational import, the object of which is to relate the biological sciences directly to human life and culture. This movement has proceeded farthest in the new modern schools, and with sixthform classes in the grammar schools. In each of these cases the topics dealt with depend largely on the interests of the teacher and his class. With no examination restrictions, presentation may sometimes be amateurist and standards rather low ; but, at any rate, links of understanding are being established between work in school and some issues in the outside world which are of major significance. 